\title{
Two fast temperature sensors for probing of the atmospheric boundary layer using small remotely piloted aircraft (RPA)
}

\author{
N. Wildmann, M. Mauz, and J. Bange \\ Center for Applied Geoscience, Eberhard-Karls-Universität Tübingen, Tübingen, Germany \\ Correspondence to: N. Wildmann (norman.wildmann@uni-tuebingen.de) \\ Received: 15 February 2013 - Published in Atmos. Meas. Tech. Discuss.: 28 March 2013 \\ Revised: 10 July 2013 - Accepted: 17 July 2013 - Published: 22 August 2013
}

\begin{abstract}
Two types of temperature sensors are designed and tested: a thermocouple and a fine wire resistance thermometer. The intention of this study is to figure out which kind of measurement principle is in general more suited for atmospheric boundary layer meteorology with small remotely piloted aircraft (RPA). The sensors are calibrated in a NIST traceable climate chamber and validated in flight against tower measurements, radiosondes and remote sensing. The sensors have a measurement range of at least $-10-50{ }^{\circ} \mathrm{C}$, an absolute RMS error of less than $\pm 0.2 \mathrm{~K}$ which is stable over the lifetime of the sensors, and a resolution of about $0.01 \mathrm{~K}$. Both devices are tested for typical errors like radiation error and adiabatic heating, as well as for their dynamic response. Spectral resolutions of up to approximately $10 \mathrm{~Hz}$ can be obtained with both sensors, which makes them suitable for turbulence measurement. Their low cost of less than 100EUR in pure hardware is a major advantage for research with small RPA.
\end{abstract}

\section{Introduction}

In situ observations in the atmosphere are of highest interest for weather nowcasting, forecasting, avionics and fundamental research. Measuring with aircraft has been a field of research for as long as aircraft exist (Neisser et al., 2002). A good overview of systems and strategies for airborne measurement of wind, temperature, and many other quantities is given in Lenschow (1986) and other specialist literature (Wendisch and Brenguier, 2013; Emeis, 1995). An important aspect for the energy budget of the earth includes the turbulent fluxes and turbulent structures of the atmospheric boundary layer (ABL) (Garratt, 1992). For the sounding of the atmosphere, numerous different methods are available nowadays: ground-based measurements, tower measurements, vertical soundings with radiosondes or tethered balloons, aircraft and helicopter measurements as well as remote sensing with $\operatorname{radar}^{1}$, $\operatorname{lidar}^{2}$, sodar ${ }^{3}$ and RASS $^{4}$, to mention only the most common instruments (Kaimal and Finnigan, 1994). Especially for the measurement of spatial averages of fluxes over heterogeneous terrain, groundbased, tower and radiosonde measurements are not suitable, because they are spot measurements which rely on the assumption of terrain homogeneity to calculate fluxes (Bange et al., 2006). Therefore, aircraft are much better suited instruments for these kinds of measurements. They can cover a wide area and perform in situ measurements that do not rely on model assumptions of the atmosphere like remote sensing instruments do.

In contrast to stationary temperature measurements, sensors in airborne applications are facing specific requirements due to their operation on a moving platform in high stream velocities. While some issues like robustness or electromagnetic compatibility have to be addressed in the design process, other error sources like the heating of the sensor due to the conversion of kinetic energy to heat at the sensor surface have to be considered in the data analysis. The most commonly used temperature sensor for airborne research is the Rosemount total air temperature sensor (Rosemount, 1986). It has been used in many meteorological campaigns with manned aircraft and systematically investigated. For example, Friehe and Khelif (1992) tested the sensor against an

\footnotetext{
${ }^{1}$ Radio detection and ranging

${ }^{2}$ Light detection and ranging

${ }^{3}$ Sound/sonic detection and ranging

${ }^{4}$ Radio acoustic sounding system
} 
NCAR K sensor and a modified Rosemount probe to find out about time responses, adiabatic heating effects and recovery factors. Inverarity (2000) found methods to correct the dynamic system of the Rosemount probe to measure more precise vertical profiles. Haman et al. (1997) designed a new sensor called "ultra-fast thermometer", which design-wise makes it possible to measure in clouds without contamination of the measurement wire with cloud droplets. It uses a $2.5 \mu \mathrm{m}$ platinum tungsten wire, which makes it fast enough to make time response corrections obsolete. All these sensors are designed to be installed on manned aircraft, and they are not optimized for small size or light weight.

Smaller sensors for airborne temperature measurement can be found in the context of radiosondes for vertical sounding of the atmosphere up to $30 \mathrm{~km}$. These sondes need to be lightweight to be able to rise and be of low cost, because once released it is not guaranteed that the instrumentation can be recovered. Commercially available sondes work with thermistors or capacitive wire technology for temperature measurement (Nash et al., 2005). Typically, the wires used in these commercial sensors are coated or protected in some way to make them more robust at the cost of response time. Correction algorithms can help to compensate the time response error (Luers, 1997). Radiosondes are not suitable for turbulent flux measurements, because they only rise vertically. At ascent rates below $10 \mathrm{~m} \mathrm{~s}^{-1}$ and time constants $\leq 1 \mathrm{~s}$, the blur in the allocation of the temperature reading to the corresponding altitude has a size of $10 \mathrm{~m}$. For their purpose of vertical soundings up to a few kilometres, this is an acceptable error.

While meteorological measurements with manned aircraft and radiosondes are frequently performed, measurements with small unmanned systems are relatively new and not well established so far. Nevertheless, numerous different systems have been developed within the last decade and have proved to produce valuable data (Martin and Bange, 2013; Martin et al., 2011; Reuder et al., 2009; Spiess et al., 2007; Dias et al., 2012; van den Kroonenberg et al., 2008). In general, the possibilities for meteorological measurements with small remotely piloted aircraft (RPA) are in between manned aircraft measurements and radiosonde soundings. Modern autopilots make it possible to fly predefined paths, even take off and land automatically if desired. Compared to manned aircraft, their cruising speed is typically lower $\left(20-30 \mathrm{~m} \mathrm{~s}^{-1}\right)$. The security standards are not as high as with manned aircraft, which in general makes them a more flexible tool, but the lack of harmonized rules in aviation authorities can cause additional limitations for flight permissions. Their application off-road makes it more likely that rough landings will damage a sensor. The space and payload capabilities are rather comparable to a radiosonde than a large research aircraft.

This work gives a description of two kinds of temperature sensors for the application on small unmanned aircraft for boundary-layer meteorology. One sensor is based on a thermocouple, while the other one is a fine wire platinum resistance thermometer (FWPRT). Both sensors were calibrated and tested in a laboratory before doing in-flight measurements and comparison of the results with stationary measurements from a $99 \mathrm{~m}$ meteorological tower, as well as remote sensing measurements from a sodar and wind profiler. The goal is to allow for measurements of the atmospheric temperature with a reasonable accuracy and sufficient resolution to also resolve turbulent fluctuations with both kind of sensors. By describing the advantages, disadvantages and problems in design and application, a guidance is given towards finding the best suitable sensor for individual RPA applications. It will be shown that these kinds of sensors can be built at low cost and that typical errors for temperature measurement can be avoided or corrected.

\section{Sensor design}

\subsection{Requirements}

The temperature sensors described here are designed for the purpose of measuring mean temperature in the ABL, as well as turbulent heat fluxes. As the target value of absolute accuracy in mean measurements, $\pm 0.1 \mathrm{~K}$ is strived for. Although this accuracy is not critical for the evaluation of thermal stratification or turbulent transport, it can still be of relevance if complementary measurements with ground-based, remote sensing or other airborne instruments are performed. A higher absolute accuracy than $\pm 0.1 \mathrm{~K}$ is hardly achieved by commercial instruments, including the reference instrument in the used calibration chamber. Therefore this value is chosen as the target value for the new sensors.

Typical values for temperature fluctuations in different regimes are given for a field experiment in Wangara (Stull, 1988), stating that in a convective regime, fluctuations $\sigma_{\theta}<1 \mathrm{~K}$ are to be expected, and in the stable regime the fluctuations are much smaller $\left(\sigma_{\theta}<10^{-2} \mathrm{~K}\right)$. From this example alone it can already be seen that it is important to measure temperature with a resolution and precision equal to or better than $0.01 \mathrm{~K}$.

Short response times are important to measure as far as possible into the inertial subrange of turbulence (Kolmogorov, 1941) to minimize the error in flux measurement introduced by neglecting small-scale turbulence. In the boundaries of solid-state temperature sensors that are robust enough for the application on small RPA, a response time smaller than $0.05 \mathrm{~s}(20 \mathrm{~Hz}$ temporal resolution) is considered a target value. Table 1 gives an overview of these requirements.

\subsection{Interfaces and data collection}

Both sensors that are described here were developed specifically for the application on a small RPA. The measuring system they are connected to is called AMOC (Airborne 
Table 1. Summary of requirements for temperature sensors for the determination of turbulent heat fluxes.

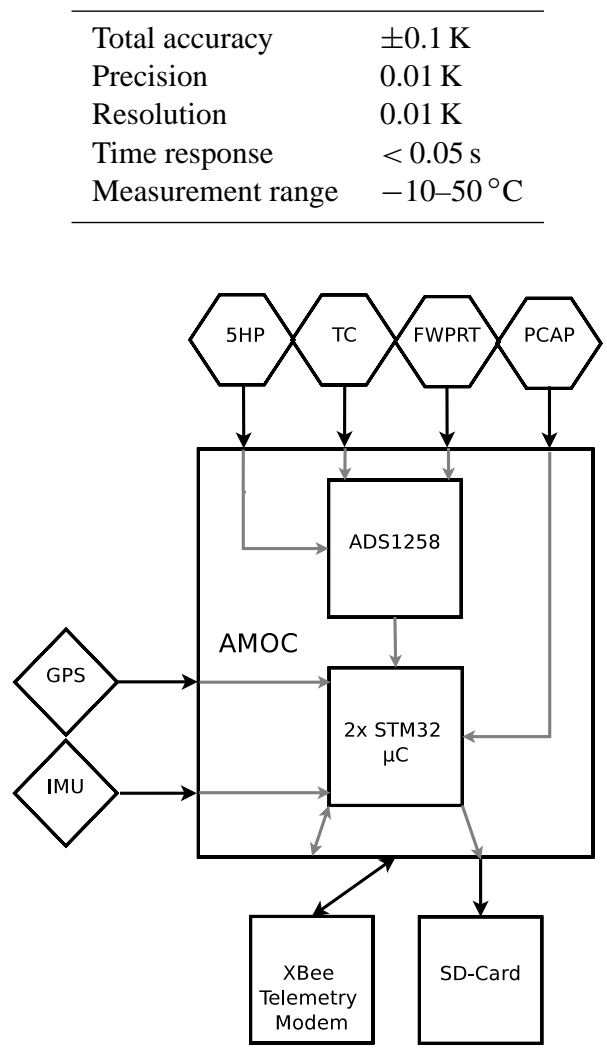

Fig. 1. Block diagram of the meteorological measuring unit for small RPA of type MASC (Multipurpose Airborne Sensor Carrier), developed at the University of Tübingen.

Meteorological Onboard Computer) and was developed in cooperation with the University of Applied Sciences Ostwestfalen-Lippe. The computer is based on two microcontrollers $(\mu \mathrm{C})$ of type STM32 and interfaces to an inertial measurement unit (IMU) and a GPS receiver. To be able to connect various other sensors, including the two temperature sensors (thermocouple, TC, and FWPRT) described here, a 16-channel analog-to-digital converter (type ADS1258 by Texas Instruments) with 24-bit resolution (15-bit noise-free) and an input range of $0-5 \mathrm{~V}$ is integrated. All data are stored on a micro-SD card with a sampling rate of $100 \mathrm{~Hz}$ and are also transmitted to a ground station computer for live observation at a rate of $1 \mathrm{~Hz}$. In standard configuration, the whole measuring unit also includes a combined humidity and temperature probe (PCAP) and a five-hole probe (5HP) to measure the true airspeed vector (van den Kroonenberg et al., 2008). A block diagram of the system is given in Fig. 1.

\subsection{Thermocouple}

Measurements with a thermocouple are in the first place always differential measurements between the measurement

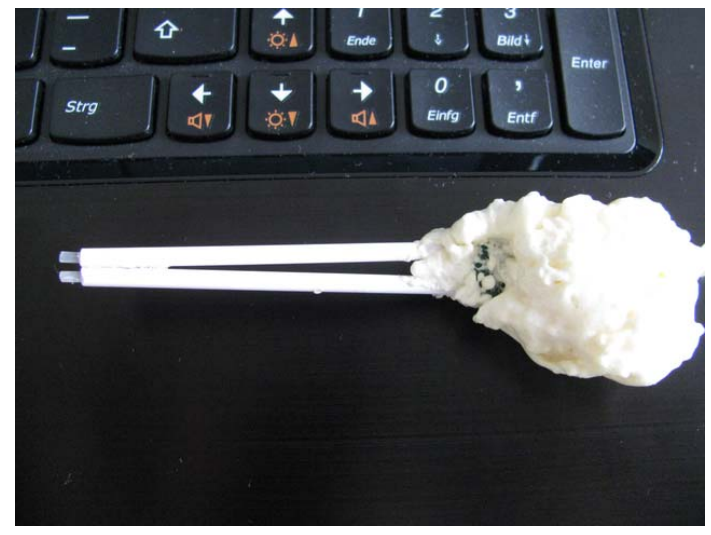

Fig. 2. Thermocouple design with foam coat for thermal insulation of the cold junction.

junction, where the thermocouple wires of unequal alloys are welded together, and the point where the thermoelectric voltage is amplified, or converted to a digital signal. Ideally this point should be in thermal equilibrium with the point where the thermocouple wires are physically connected to the electronics, in order not to measure temperature gradients in the measuring system. The big advantage of this method is the possibility to manufacture thin, long thermocouple wires to make point measurements of temperature in remote locations with a very short response time. To get the true absolute temperature, the differential measurement of the thermocouple always has to be added to a measurement of the cold junction temperature (Michalski et al., 1991). Therefore, a welldefined cold junction of the sensor is essential. A possibility to account implicitly for the cold junction temperature is to generate a voltage at the cold junction that corresponds to the thermoelectric voltage of the chosen thermocouple type for a temperature difference of the actual cold junction temperature. This voltage can then be added to the thermoelectric voltage of the thermocouple itself in an analog circuit, which after amplification and multiplication with the correct calibration coefficients gives an absolute temperature reading. An integrated circuit which takes care of this is the LTK001 by Linear Technology. This chip is used in the design shown in Fig. 2.

For this set-up to work, it is essential that the temperature sensor inside the LTK001 measures the true temperature of the cold junction. If there are gradients between soldering junction of the thermocouple wire and the chip, or if the temperature sensor inside the chip responds too slow to a sudden change in temperature, the temperature reading can be corrupted. To reduce these effects, the whole electronic components including the thermocouple connection were coated in insulating foam (Fig. 2).

Another method to get even more accurate absolute temperature readings from the thermocouple is a complementary filter. This means the thermocouple signal is high-pass 


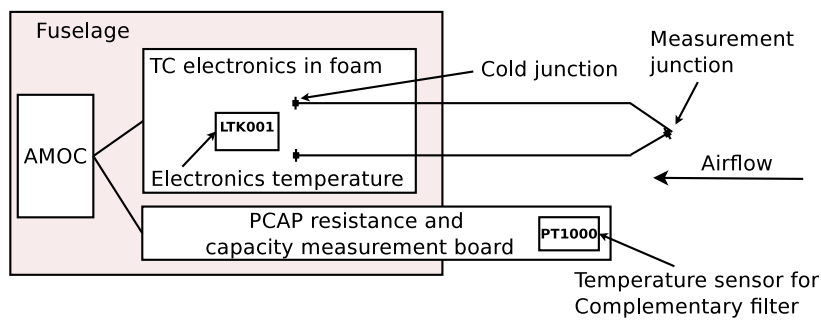

Fig. 3. Schematic drawing of the complete thermocouple measurement strategy.

filtered and added to a signal of a complementary temperature sensor that has a low-pass filter with the same cut-off frequency as applied for the high-pass of the thermocouple. The complementary sensor has to be a more stable and accurate temperature sensor that is also in the flow and responds just fast enough to sense the frequencies that are corrupted by wrong cold junction readings of the thermocouple. For this purpose a PT1000 was installed in close distance to the thermocouple on the RPA. The resistance of the PT1000 is measured with the PCAP01 signal processor by ACAM Messelectronic, which is also responsible for the measurement of a capacitive humidity sensor. This combined sensor is referred to as PCAP in this article. Figure 3 shows a sketch of all the sensors and temperatures involved in the current design. The thermocouple was built and tested with two different wire diameters $(25 \mu \mathrm{m}$ and $13 \mu \mathrm{m})$. Like in other meteorological applications (Haman, 1992), the thermocouple type $\mathrm{E}$ (CHROMEGA ${ }^{\circledR}$ chromium nickel alloy and Constantan ${ }^{\circledR}$ ) was chosen due to its chemical resistance and high sensitivity $\left(60.9 \mu \mathrm{V} \mathrm{K}^{-1}\right)$.

For all measurement results of the thermocouple in the following, the complementary filtered signal is used. It showed the more trustworthy results compared to the internal coldjunction compensation of the LTK001. The amplification of the pure thermocouple voltage is chosen to achieve a sensitivity of $20 \mathrm{mV} \mathrm{K}^{-1}$. Typical noise level for the whole measuring chain is $0.15 \mathrm{mV}$, so a resolution of $7.5 \mathrm{mK}$ is realistic. The measuring range is adjusted to $-10-240^{\circ} \mathrm{C}$. The precision and stability of the operational amplifiers that are used in the design is in the $\mu \mathrm{V}$ range, which is orders of magnitude better than the required precision.

\subsection{Fine wire platinum resistance thermometer (FWPRT)}

The FWPRT was designed based on Harrison and Pedder (2001). Few adaptations were done in the choice of operational amplifiers, and a printed circuit board was designed to reduce noise and size of the set-up. The basic principle of the circuit is a linearized Wheatstone bridge with current output, which is in the final step amplified and converted to a $0-5 \mathrm{~V}$ signal corresponding to approximately -10 to $50^{\circ} \mathrm{C}$ measuring range. The measuring range can easily be adapted by

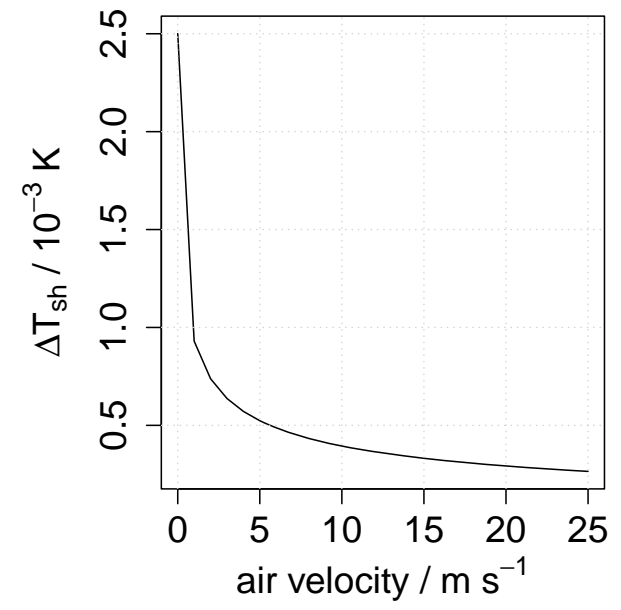

Fig. 4. Theoretically calculated self-heating for a $25 \mu \mathrm{m}$ platinum wire with the designed measuring circuit.

the choice of the reference resistor in the Wheatstone bridge. One source of error that can already be addressed in the design process is self-heating of the fine wire. The bridge is designed and simulated to have a measuring current through the platinum wire of $0.6 \mathrm{~mA}$. If we take into account that we have forced convection on the wire in flight, we can set up a power budget $P_{\mathrm{I}}=P_{\mathrm{c}}$ which relates the power introduced by the measuring current $P_{I}=R \cdot I^{2}$ and the convective heat loss $P_{\mathrm{c}}=\alpha \cdot A \Delta T_{\mathrm{sh}}$ to calculate the maximum self-heating of the wire:

$\Delta T_{\mathrm{sh}}=\frac{R \cdot I^{2}}{\alpha \cdot A}$,

where $\Delta T_{\mathrm{sh}}$ is the temperature change due to selfheating, $R=100 \Omega$ the resistance of the platinum wire, $I=0.6 \times 10^{-3} \mathrm{~A}$ the measuring current, $\alpha$ the heat transfer coefficient calculated from the Nusselt number $\left(0.39+0.51 \cdot R e^{0.5}\right.$, with $R e=$ Reynolds number $)$ and the molecular thermal conductivity of the air at a specific airstream velocity, and $A=3.7 \times 10^{-5} \mathrm{~m}^{2}$ the curved surface area of the wire (Foken, 1979). Figure 4 shows the theoretical self-heating for these values over a range of airspeeds calculated with Eq. (1). It can be seen that for this measuring current, self-heating effects are two orders of magnitude smaller than the goal for measurement accuracy and thus can be neglected.

The platinum wire of 25 and $13 \mu \mathrm{m}$ respectively is wrapped around the printed circuit board with minimum contact to the board itself, as can be seen in Fig. 5. It can also be seen that the wires are aligned in flight direction, which will theoretically minimize the effect of adiabatic heating of the wire. The resistance value of $100 \Omega$ is a good compromise considering resolution, wire length and self-heating. Reducing wire length to get closer to point measurements will also reduce the resistance. A higher resistance gives better resolution, but the measuring current will necessarily increase, 


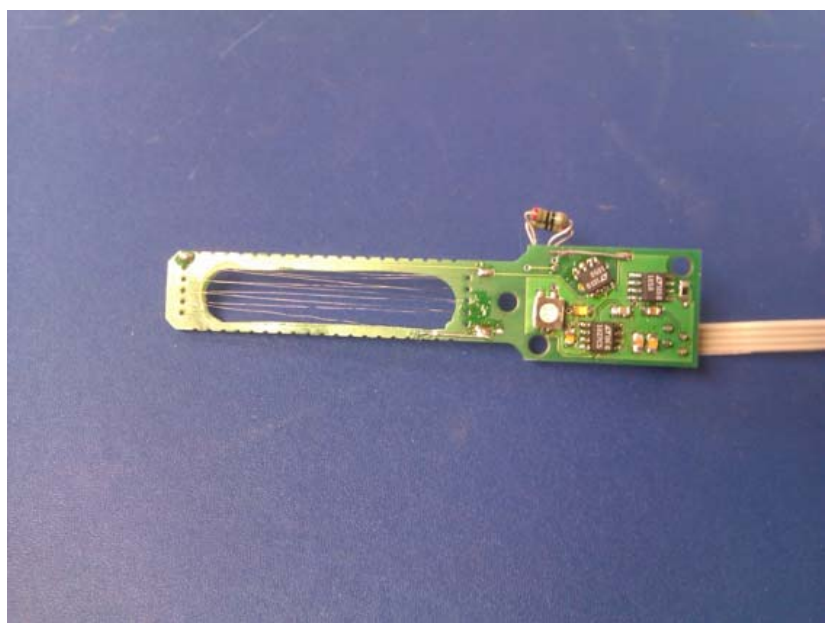

Fig. 5. Fine wire platinum resistance thermometer with $25 \mu \mathrm{m}$ wire. The flow direction is from the left.

which leads to higher self-heating. The sensor has a sensitivity of $80 \mathrm{mV} \mathrm{K}^{-1}$ for a measuring range of $-10-50^{\circ} \mathrm{C}$. The noise level is equivalent to the thermocouple circuit, so that a resolution of $1.8 \mathrm{mK}$ is reached. Like in the thermocouple circuit, operational amplifiers were chosen that have a gain stability and precision that outranges the requirements.

\section{Validation flights}

\subsection{The test site}

An experiment was performed in September 2012 at a test field of the German Meteorological Service (Deutscher Wetterdienst - DWD) close to Lindenberg, Germany. The site is located at $52.1669^{\circ} \mathrm{N}, 14.1242^{\circ} \mathrm{E}$ in north-east Germany in a heterogenous but nearly flat terrain. It was used previously in comparative sensor studies for airborne meteorology (Martin et al., 2011). Approximately $200 \mathrm{~m}$ from the take-off location for the RPA, a $99 \mathrm{~m}$ tower with meteorological instruments at 6 heights, including temperature, humidity, wind speed and wind direction measurements, is installed. The tower is also equipped with sonic anemometers at two levels $(50$ and $90 \mathrm{~m})$ for turbulence measurements. Besides the tower, there are also various remote sensing instruments installed on the test field. For comparison of temperature measurements, a sodar/RASS and a $482 \mathrm{MHz}$ wind profiler/RASS can be taken into account, which both measure virtual temperature of the atmosphere along with wind speed and wind direction (Engelbart et al., 1999; Engelbart et al., 1996; Engelbart and Bange, 2002). The sodar is installed directly at the test site, while the wind profiler is situated at the observatory in Lindenberg, $5 \mathrm{~km}$ north of the site. Additionally, every $6 \mathrm{~h}$, a radiosonde is released in Lindenberg for soundings of temperature, humidity and wind.

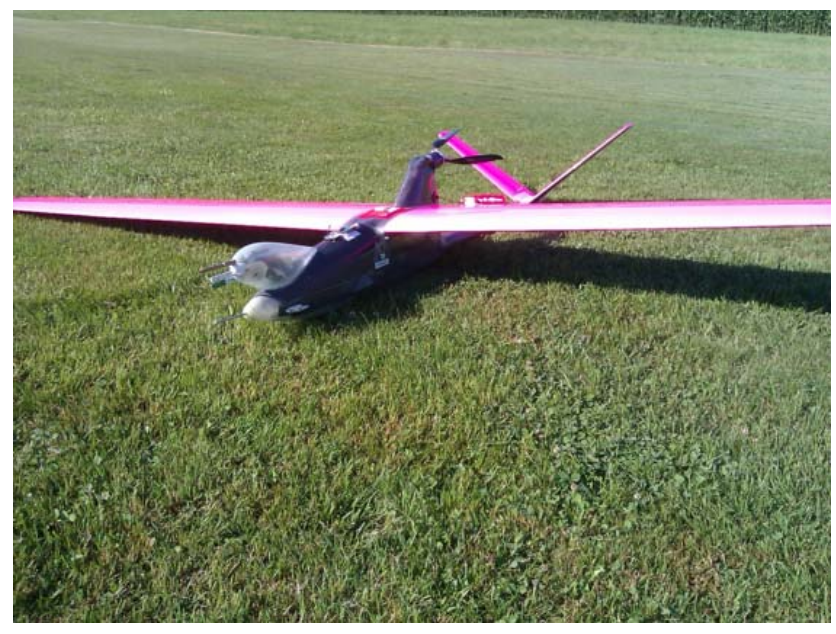

Fig. 6. MASC airframe.

\subsection{The RPA system MASC}

The platforms that are being used at the University of Tübingen to perform meteorological measurements are small RPA named MASC (Multi-purpose Airborne Sensor Carrier), which were developed in-house in cooperation with a local model aircraft builder. The MASC airframe as shown in Fig. 6 is an electrically powered motor glider aircraft of $3.00 \mathrm{~m}$ wingspan and a maximum take-off weight of $6 \mathrm{~kg}$. In standard configuration with meteorological payload, the weight does not exceed $5.5 \mathrm{~kg}$. A flight endurance of at least $40 \mathrm{~min}$ is possible at this weight with minimum battery load, which corresponds to a travelling distance of more than $50 \mathrm{~km}$ at a travelling speed of $22 \mathrm{~m} \mathrm{~s}^{-1}$. Tests in the wind tunnel showed that the pusher motor does not affect the measurement system at the fuselage tip. Only very little flow disturbance was found due to the fuselage itself at typical airspeeds.

The aircraft are equipped with the autopilot ROCS (Research Onboard Computer System), which was developed at the Institute of Flight Mechanics and Control (IFR) at the University of Stuttgart (Haala et al., 2011). It was originally developed for photogrammetric applications and specifically adapted to the needs of meteorological observations. The autopilot makes it possible to fly pre-defined flight patterns automatically, guaranteeing a constant airspeed of $22-24 \mathrm{~m} \mathrm{~s}^{-1}$ and a level flight with a precision in altitude better than $2 \mathrm{~m}$ in straight legs. A bias in altitude can be up to $10 \mathrm{~m}$.

\subsection{The experiment}

The experiment was carried out in autumn 2012 from 21 to 23 September. Two MASC systems were operated in this period. While on the first and third day of the experiment fair weather allowed a total number of 15 measurement flights with the system, the second day with more gusty winds and light rain was only used for test flights and technical 

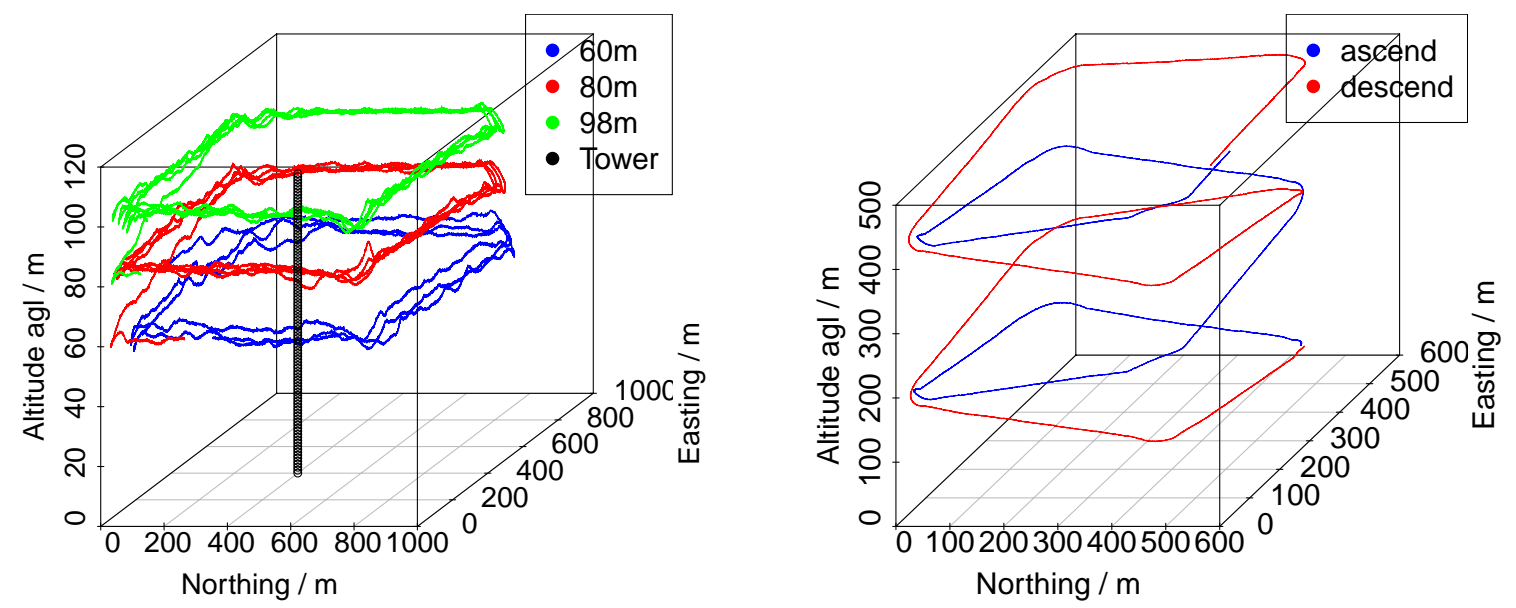

Fig. 7. Flight paths for tower sweep maneuver (left panel) and vertical profiles (right panel). The solid black vertical line represents the measurement tower.

improvements on the system. Several flights were done specifically to validate the temperature measurements of the two sensors described here. Two flights were performed flying squares with a size of $600 \mathrm{~m}$ around the tower at three heights where sensors are installed on the tower $(60,80$ and $98 \mathrm{~m})$. Another two flights were done at the time when radiosondes were released to measure vertical profiles between 60 and $500 \mathrm{~m}$ and compare the results. Figure 7 shows the flight paths for these two maneuvers. For comparison with the tower, the aircraft remained at each altitude for 8 to $10 \mathrm{~min}$ to have the same averaging period as the tower data. During straight and level flights, the standard deviation of barometric altitude is below $0.5 \mathrm{~m}$. Including the bends, it is still below $2 \mathrm{~m}$. Lateral deviations from the given track are within $\pm 2 \mathrm{~m}$. For the vertical profile, a constant climb rate of about $2 \mathrm{~m}$ per second was chosen.

All temperature sensors involved were calibrated in a NIST (National Institute of Standards and Technology, USA) traceable climate chamber for a range of $15^{\circ} \mathrm{C}-50^{\circ} \mathrm{C}$. In the experiment, temperatures slightly below the calibration range were experienced. However, subsequent calibrations of the same type of sensors to lower temperatures showed that the sensor output follows the calibration curve very well down to $10^{\circ} \mathrm{C}$ and further, staying well within the desired accuracy of $0.1 \mathrm{~K}$.

\subsection{Results}

The results of the comparison flights with the tower at three levels can be seen in Fig. 8. All flights show that the FWPRT measures a temperature that is constantly higher than the temperature measured at the tower. The two flights on 21 September were done with exactly the same FWPRT sensor; on 23 September another FWPRT sensor of the same type was used. The sensor used on 23 September has a slightly bigger offset of about $0.6 \mathrm{~K}$, compared to
$0.4 \mathrm{~K}$ on 21 September. The PCAP sensor that is being used for filtering with the thermocouple systematically shows a lower measurement of about $0.2 \mathrm{~K}$ compared to the tower. These constant errors can be interpreted as calibration errors and are easily corrected by subtracting the measured offset. Analysing all 10-minute averages that were possible to extract from airborne measurements throughout the experiment, it was found that a mean error of $-0.26 \mathrm{~K}$ with a standard deviation of $0.1 \mathrm{~K}$ for the PCAP sensor and a mean error of $0.46 \mathrm{~K}$ with a standard deviation of $0.09 \mathrm{~K}$ for the FWPRT were measured (see Fig. 9). The requirement of $0.1 \mathrm{~K}$ hence can be held throughout the experiment, if a calibration offset between the sensors is subtracted once. The random errors include possible errors due to a bias between tower instrument height and controlled aircraft altitude, which can be up to $10 \mathrm{~m}$. An error in altitude of this order at a lapse rate of $0.01 \mathrm{~K} \mathrm{~m}^{-1}$ will result in a temperature error of $0.1 \mathrm{~K}$, which is in the range of the standard deviation. Another possible error source is the fact that the aircraft measurement is a spatial average, while the tower measurement is a point measurement. In laboratory conditions in a climate chamber, the sensors never exceed $0.1 \mathrm{~K}$ deviation with a quadratic fit over the whole calibration range. The experiment in Lindenberg could only cover a small range of temperatures. To find out if the errors of the sensors are larger close to the edges of the calibration range or even beyond, more flight tests are needed.

Figures 10 and 11 show the result of the temperature sensors for two sequential vertical profiles at around 11:00 UTC and around 17:00 UTC respectively, in comparison to all other temperature measurements that were done at the test site in Lindenberg. For better comparison with the remote sensing systems, the RPA, radiosonde, and tower measurements are converted to virtual temperature according to the equations in Appendix A. The measurement of relative humidity was done with a capacitive sensor, which was found 

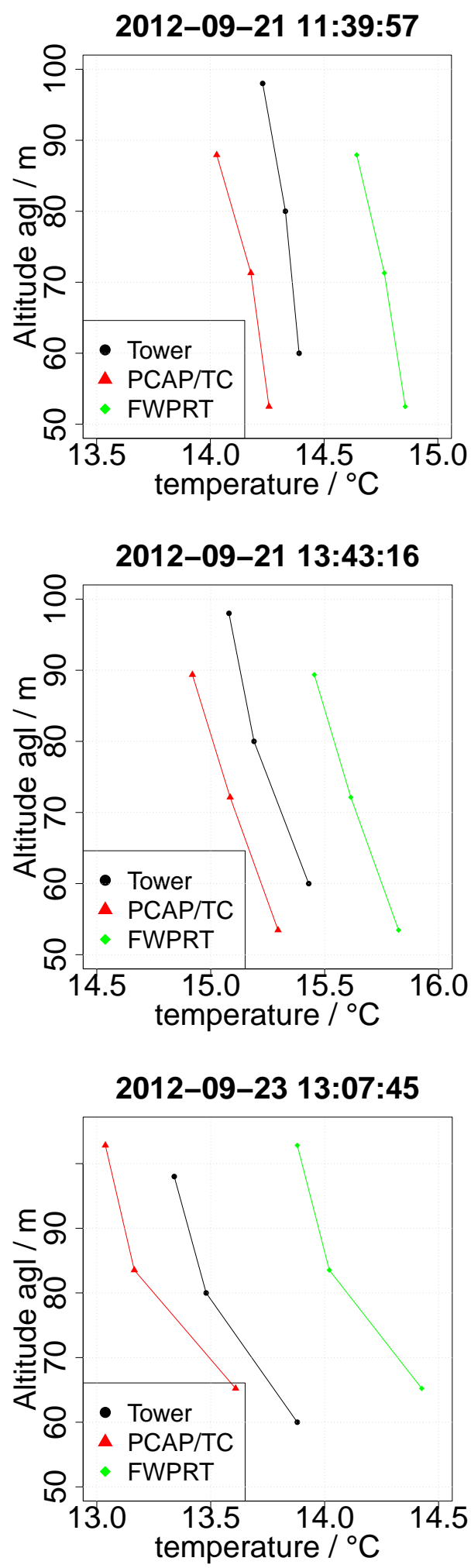

Fig. 8. Comparison of temperature sensors on the RPA with tower data, 10 min averages at three levels and three different flights. to be in good agreement with the reference instruments in this experiment. Deviations of up to $\pm 5 \% \mathrm{RH}$ can be observed, which, according to the conversion to virtual temperature, can cause temperature errors of maximum $\pm 0.05 \mathrm{~K}$ for the given situation. Looking at all profiles, it can be seen that, for the vertical profiles as well, the FWPRT has an offset to the tower of about $0.5 \mathrm{~K}$. The offset to the radiosonde and the wind profiler is in the same range. For the FWPRT, the lapse rate of virtual temperature is very well captured by the sensors, and ascents and descents do not show remarkable hysteresis, which shows that the sensor's time response is fast enough for the given climb rate. At around 11:00 UTC, the radiosonde measures a higher lapse rate close to the ground which is captured neither by the tower, nor by the RPA. In the late afternoon measurement, RPA, tower and radiosonde profiles have the same shape down to the ground in consideration of the different ground levels. Most probably a local effect in the area where the radiosonde was released, which is approximately $5 \mathrm{~km}$ from the $99 \mathrm{~m}$ tower, is the reason for this difference in shape close to the ground between the measurements at around 11:00UTC. Thermocouple measurements (Fig. 11) show a strong hysteresis, which is due to the PCAP sensor, which is not able to adapt fast enough to temperature changes for the given climb rate, and the thermocouple's reference junction is not stable enough to make it possible to fill the gap to lower frequencies with pure thermocouple measurements.

\section{Discussion of measurement errors}

Two of the error sources that are most cited when it comes to temperature measurement with aircraft are radiation errors and errors due to adiabatic heating of the sensor element (Breitkopf and Kim, 1980; Foken, 1979; Daniels, 1968; Shannon and Butler, 2003). The measurement campaign in Lindenberg was also used to investigate these errors for the two sensor types described above. Condensation or icing, which both have fatal effects on measurements with fine wires, will not be considered.

\subsection{Radiation error}

To figure out the effect of radiation on the sensors, square pattern flights were chosen as well. In these flights, the aircraft was flying in all four main geographic directions. The flights were performed in late morning, between 10:00 and 11:00 UTC, with the sun in southern position (azimuth $\approx 174^{\circ}$, elevation $\approx 38^{\circ}$ ). The sensors are installed on the RPA in a way such that, for flights in north and east direction, sun was shining on the sensors, but in south and west direction, the sensors are shaded by the RPA itself. For FWPRT measurements, no significant offset between different flight directions could be found. For thermocouple measurements, an effect could be observed and is shown in Fig. 12 

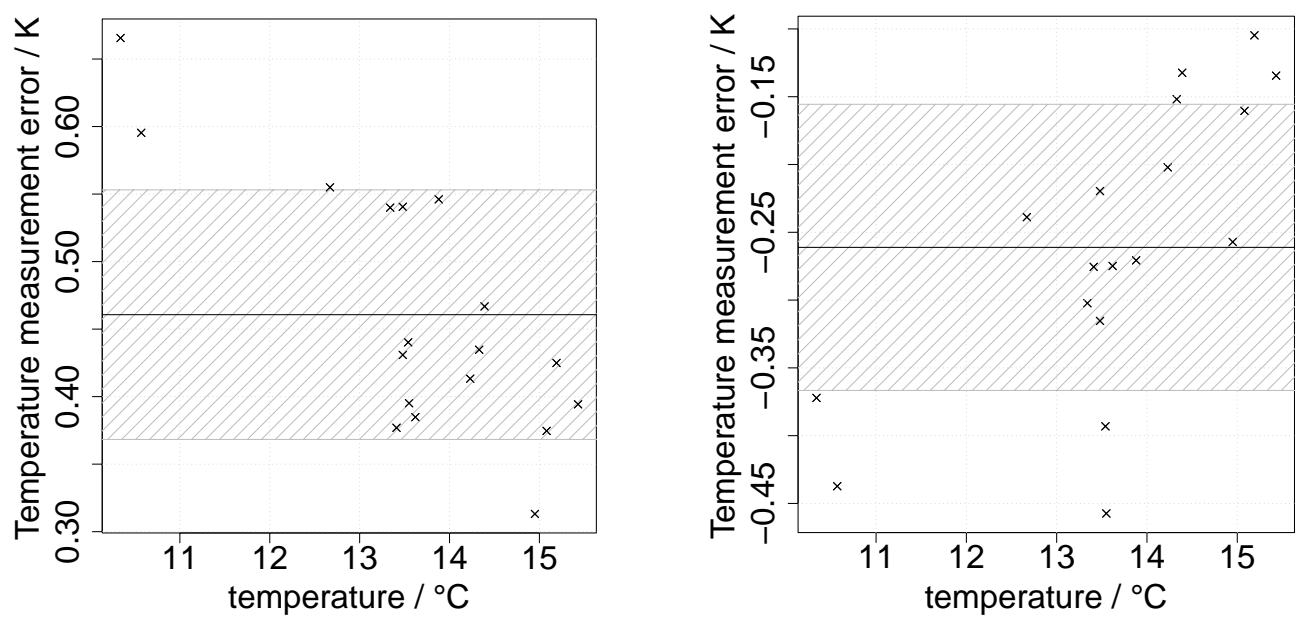

Fig. 9. Errors of FWPRT (left panel) and thermocouple measurements (right panel) compared to tower temperature measurement. Ten-minute averages of RPA and tower measurements at the same altitude and time are used to calculate the error. The solid black line marks the mean error. The shaded area visualizes the standard deviation.

FWPRT, around 11 UTC

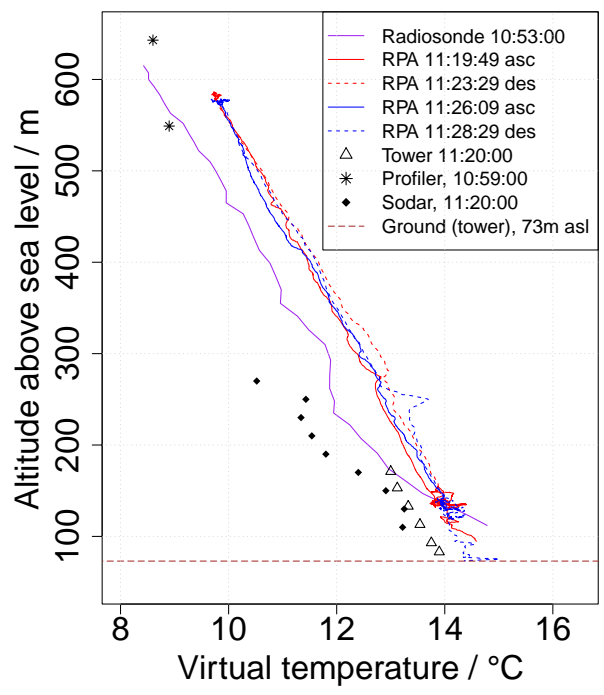

FWPRT, late afternoon

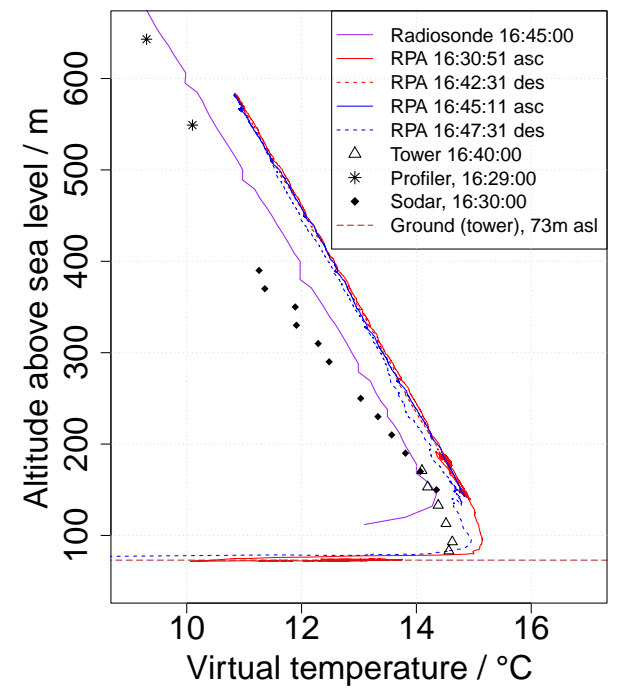

Fig. 10. Comparison of FWPRT on the RPA with remote sensing and tower data for vertical profile flights on Sunday, 23 September 2012.

(left panel). It shows mean values for each leg in the square, coloured according to the flight direction. For each square the temperature rises, but a constant offset between counterdirections of about $0.5 \mathrm{~K}$ is observed. The source for this offset is the PT1000 on the PCAP sensor. Repeating the same pattern with a shield around the PCAP sensor gives much better results with no significant offset between all directions (Fig. 12, right panel). The shield is made of a $2 \mathrm{~cm}$ diameter carbon tube covered with white adhesive film that completely covers the PCAP electronics. This result agrees with experiments performed in a wind tunnel using a light bulb as radiation source and exposing the sensor system with and without radiation shield. Both fine wire sensors - thermocouple and FWPRT - do not show a significant sensitivity to radiation at the given airspeed of $20 \mathrm{~m} \mathrm{~s}^{-1}$. This is a good indication that radiation errors can be neglected for this type of sensor. In wind tunnel experiments the radiation input was increased to a much higher level than in the test flights, radiating the sensors with up to $800 \mathrm{~W} \mathrm{~m}^{-2}$, which corresponds to a hot summer day (see Fig. 13). The times when the radiation was switched on and off can clearly be seen in the PCAP measurements, while the FWPRT reacts with less than $0.2 \mathrm{~K}$ deviation from the wind tunnel internal temperature trend.

\subsection{Adiabatic heating}

Adiabatic heating of a sensor occurs when the air is decelerated at the sensitive element of the measurement instrument. 
Thermocouple, around 11 UTC

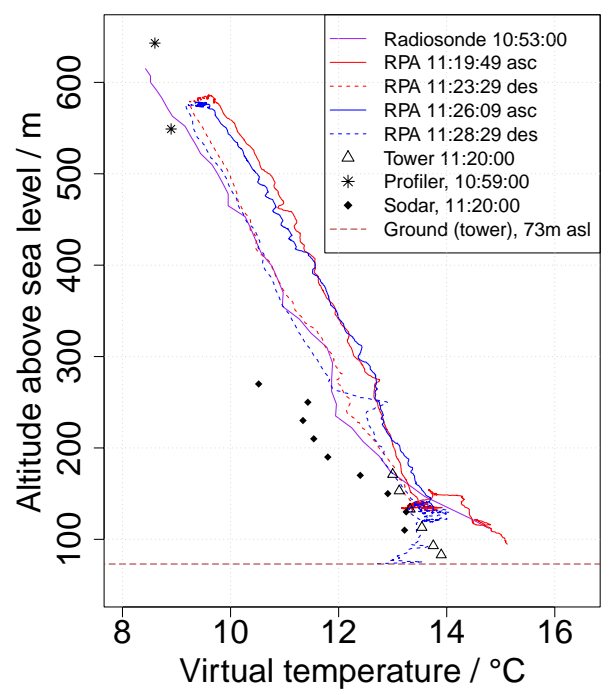

Thermocouple, late afternoon

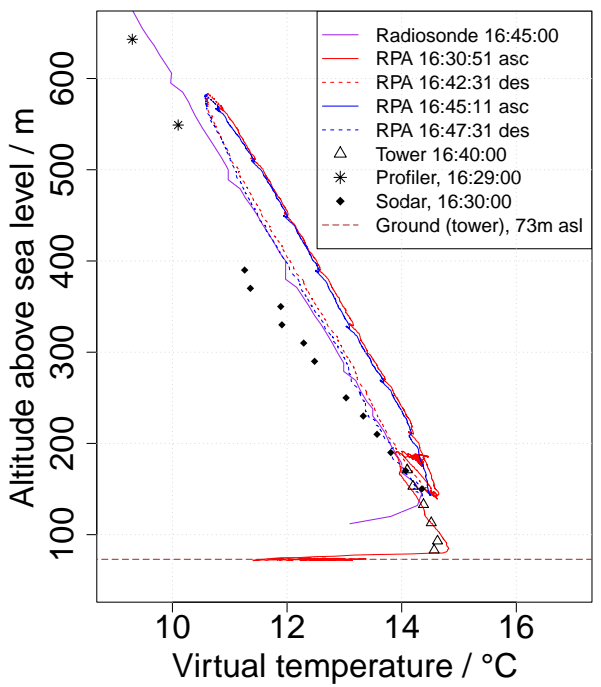

Fig. 11. Comparison of thermocouple on the RPA with remote sensing and tower data for vertical profile flights on Sunday, 23 September 2012.
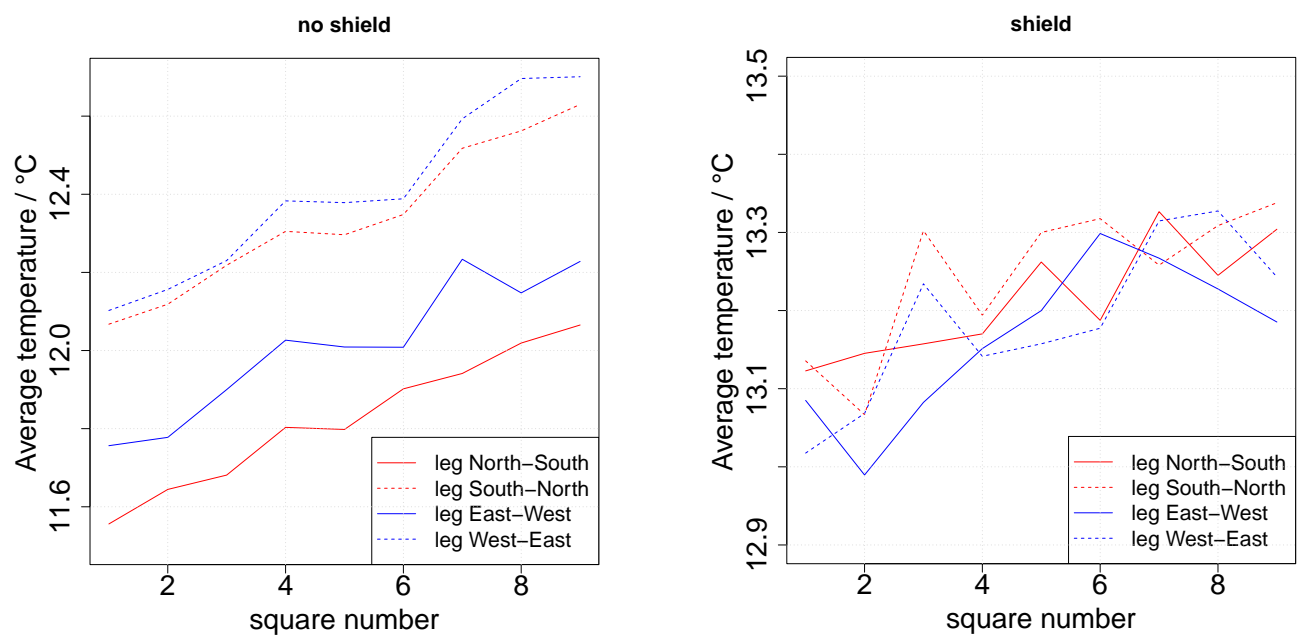

Fig. 12. Averaged thermocouple temperature measurement over legs in all geographic directions with and without radiation shield of the PCAP sensor. Dashed lines are for legs with irradiated sensors; solid lines are for legs with sensors in the shadow of the fuselage.

The kinetic energy of the air is transformed to heat. This heat leads to higher measurements compared to the static temperature. In theory, adiabatic heating can be described with the following adiabatic equation:

$T_{\mathrm{s}}=r \cdot T_{\mathrm{m}}\left(\frac{P_{\mathrm{s}}}{P_{\mathrm{t}}}\right)^{\kappa}$,

where $T_{\mathrm{S}}$ is the static temperature of the air, $T_{\mathrm{m}}$ the temperature measured by the sensor, $P_{\mathrm{S}}$ the static pressure, $P_{\mathrm{t}}$ the total pressure including the dynamic pressure at the sensor and $\kappa=R_{\mathrm{d}} / C_{\mathrm{p}} \approx 0.28571$ the Poisson constant (ratio of the gas constant and the specific heat for constant pressure). Using this equation with boundary conditions of $293 \mathrm{~K}$ measured temperature, static pressure of $1000 \mathrm{hPa}$ and an airspeed of $25 \mathrm{~m} \mathrm{~s}^{-1}$ leading to a total pressure of $1003.70 \mathrm{hPa}$, the measured temperature is $\approx 0.3 \mathrm{~K}$ higher than the true static temperature. In reality, the air is not only decelerated at the sensitive part of the sensor. The geometry of the sensor plays an important role for the effect of adiabatic heating. This is typically considered by the introduction of a recovery factor $r$. The value of this recovery factor has to be evaluated in experiments (Breitkopf and Kim, 1980). To see if the adiabatic heating effect plays a role at all for the sensor under investigation, the cross-correlation between temperature measurement and airspeed in a measurement flight at constant altitude can be calculated. Figure 14 shows the results for the 


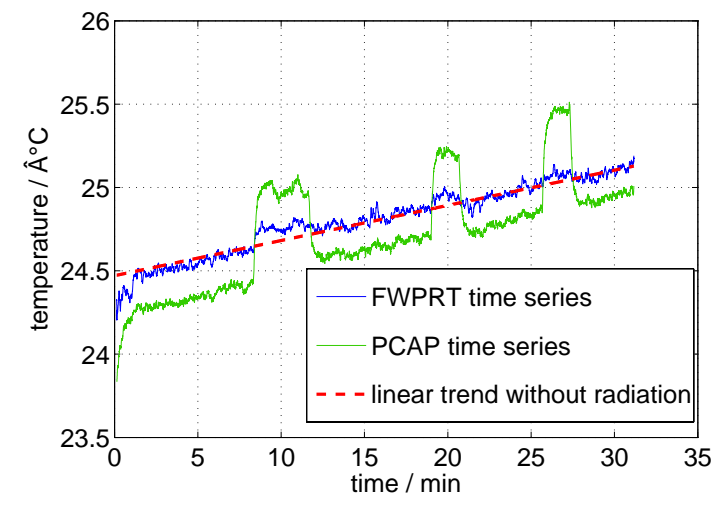

Fig. 13. A time series of a wind tunnel experiment with artificial radiation source.

FWPRT and the thermocouple. Both maximum correlation coefficients are below 0.1. This is considered small enough to neglect errors of adiabatic heating in future experiments.

\section{Spectral responses}

To measure turbulent fluxes of heat, it is important to resolve eddies as far as possible into the inertial subrange of turbulence. A spectral analysis of the measurements shows how high the temporal resolution of the sensor is, if the result is compared to the Kolmogorov law of locally isotropic turbulence in the inertial subrange (Kolmogorov, 1941). For the spectral analysis, the experiment in Lindenberg was not useable, due to internal noise caused by the telemetry system. This noise could be seen on all analog signals and was especially critical for thermocouple measurements due to the small voltage signal of this sensor. It could be clearly due to the telemetry, because it appeared at the same frequency as the downlink frequency.

Subsequent tests showed that the electronic circuit as it was used in Lindenberg also artificially limited the frequency response for the FWPRT. The current to voltage converter includes an active low-pass filter, which was adjusted to a cutoff frequency of about $5 \mathrm{~Hz}$. A trade-off needed to be found for a higher cut-off frequency while maintaining a reasonable signal-to-noise ratio at the same time.

Figure 17 shows variance spectrum and structure function of a flight with the optimized electronic circuit. In this case, the sensitivity of the sensor is reduced to $40 \mathrm{mV} \mathrm{K}^{-1}$, which still meets the requirements described in Sect. 2. It can be seen that the spectral results of both sensors agree very well, especially in the higher frequency range. The flights were done at $370 \mathrm{~m}$ altitude on a summer day in June, just before noon, in southern Germany. At this altitude, a stable stratification was still present as can be seen from a vertical profile taken just before the racetrack pattern flights for the spectral analysis (see Fig. 15). Figure 16 shows the time series of the complete racetrack pattern, including bends. In fact, very similar results have to be expected since the main physical influences to the sensor response are the wire diameter and the forced convection due to airspeed, which in this case are identical for both sensors. Additionally, the same data acquisition system and same operational amplifiers were used, so that these influences are eliminated for the comparison. Remaining differences can mainly be due to imperfect calibration and the error sources that were discussed before. Radiation, adiabatic heating and heat transfer might not contribute significantly to the absolute reading, but can still have some influence on very small scales.

The variance spectrum and structure function do not perfectly follow the Kolmogorov law of locally isotropic turbulence (inertial subrange) in this measurement. There are several possible reasons for this. Due to restrictions in the flight permission, only rather short legs of about $1 \mathrm{~km}$ could be performed. Only four legs were used for the averaging of the spectral analysis because of the instationarity of the ABL. Thus, temporal variations of the spectral density can be expected. Also, in the residual layer, turbulence is weak and not necessarily isotropic since the turbulent eddies are compressed vertically.

\section{Conclusions}

In this study, two temperature sensors for airborne flux measurements in the atmospheric boundary layer were developed and tested extensively. Section 2 introduces the requirements and explains the system design. The resolution and measuring range was designed to be met for both sensor types. Section 3 shows that each of the sensors has the ability to measure temperature within the desired accuracies, if calibration offsets are subtracted. Section 4 discussed the typical errors in airborne temperature measurements like radiation error and adiabatic heating and showed that for the given sensors these are small enough to be within the total accuracy of the sensors. Last, the spectral response of thermocouple and FWPRT were compared in Sect. 5. It showed that both sensors can resolve turbulent fluctuations with little damping up to $10 \mathrm{~Hz}$. The cut-off frequency for both sensors with the given design is at about $20 \mathrm{~Hz}$. Each sensor has certain advantages and disadvantages. While using thermocouple circuits, it is most critical to provide a well-designed cold junction and an appropriate temperature measurement of the cold-junction temperature to achieve good total accuracies. The FWPRT with same diameter needs a longer wire to achieve a good resolution. A longer sensitive part of the sensor also means higher chances for contamination and damage. In future designs of thermocouple circuits, more effort has to be taken into a well-measured cold-junction temperature to make it a stand-alone temperature sensor for both good absolute accuracy and fast response. Alternatively, the result of the thermocouple measurement can also be complementarily filtered with the FWPRT sensor. Future designs of 

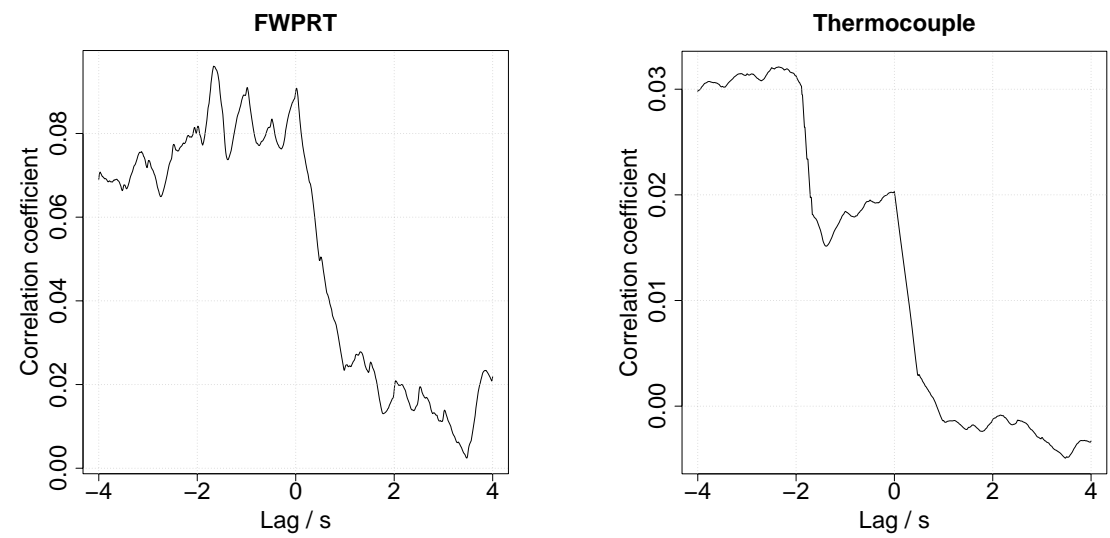

Fig. 14. Correlation function between measurements of two temperature sensors and true airspeed.

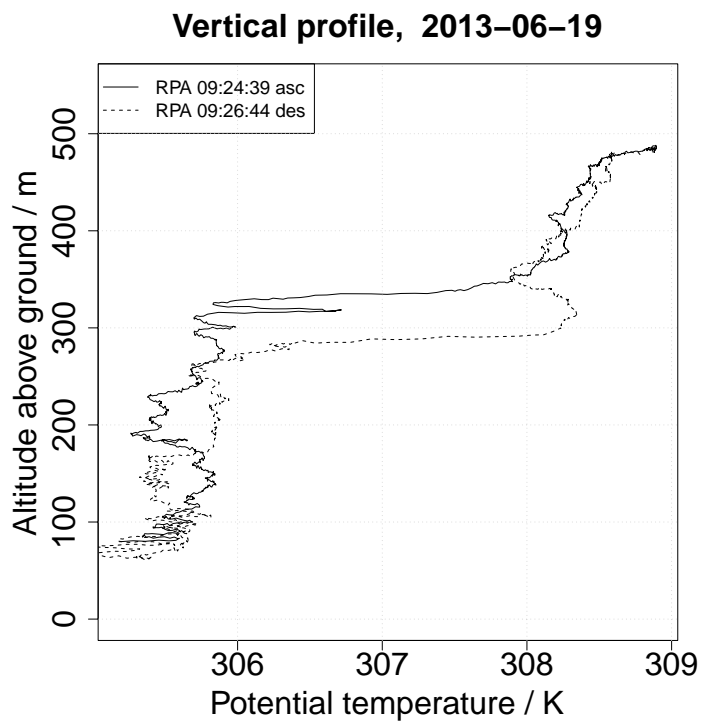

Fig. 15. Vertical profile of potential temperature measured just before the racetrack pattern for the spectral analysis of the sensors.

the FWPRT sensor could aim for better signal-to-noise ratio with smaller wire length and therefore lower resistance value of the wire. A shorter wire is less susceptible to damage in flight. This would also reduce the contact spots of the wire and thereby heat transfer to the circuit board. Heat transfer did not show to be a problem in the current frequency range, but might affect the sensor at higher frequencies. For both sensors, improvements on the electronic circuits can be done in the future to reduce the time responses as far as possible.

Each of the sensors - as they were tested in this study - have pure hardware and manufacturing costs of less than 100 EUR. Therefore, these types of sensors are the ideal equipment for fast temperature measurements aboard small RPA of type MASC or even smaller.

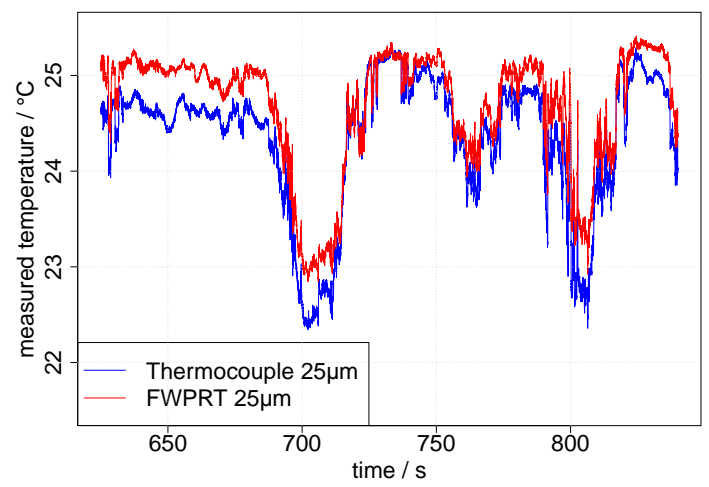

Fig. 16. Time series of thermocouple (blue) and FWPRT (red) in comparison.

\section{Appendix A}

\section{Virtual temperature}

To calculate virtual temperature from each instrument under investigation, the relative humidity and barometric pressure measurements of the respective system are used. First the saturation water vapour $E$ for the measured static temperature $T$ (in ${ }^{\circ} \mathrm{C}$ ) is calculated:

$E=6.107 \times 10^{\frac{7.45 . T}{235.0+T}} \mathrm{hPa}$.

The saturation water vapour $E$ is multiplied with the measured relative humidity value $\varphi$ to get the actual water vapour partial pressure $e$ :

$e=\frac{\varphi}{100 \%} \cdot E$.

The mixing ratio $m$ is calculated using the instrument's barometric pressure measurement $p_{s}$ and water vapour partial pressure $e$ :

$m=621.97 \cdot \frac{e}{p_{s}-e}$. 

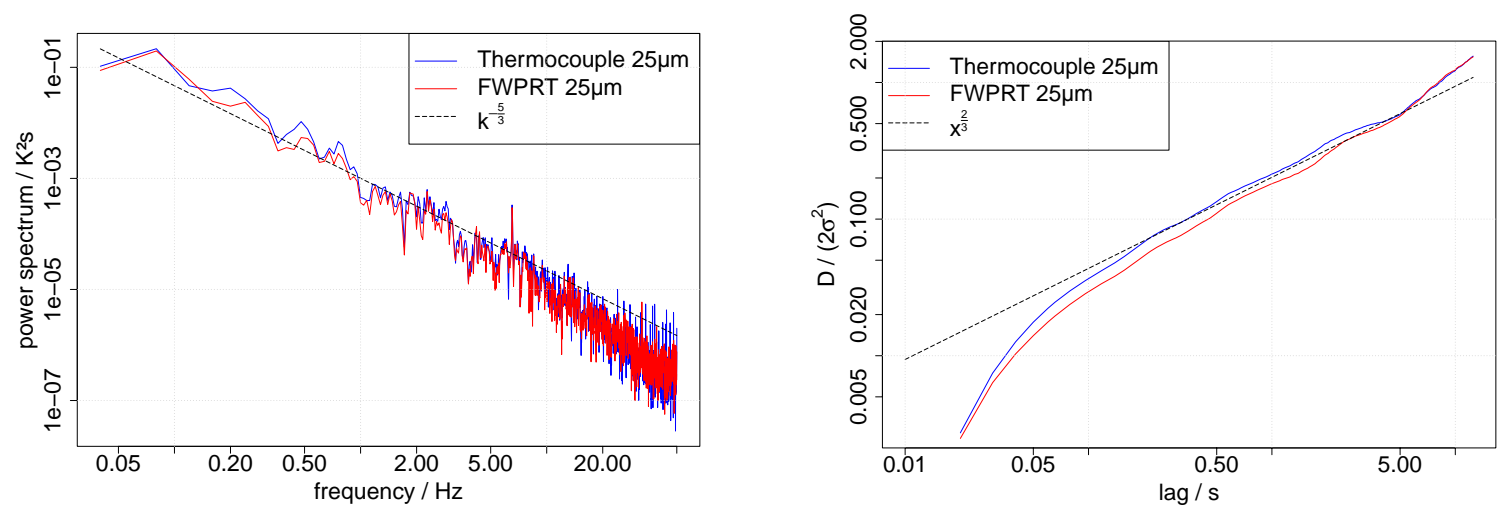

Fig. 17. Variance spectra (left panel) and structure function (right panel) of both sensors, compared to Kolmogorov laws of turbulence: the spectra that are shown are averaged over four single spectra of $25 \mathrm{~s}$ time series each. The structure function is normalized by $2 \sigma_{T}^{2}$.

Finally, the virtual temperature $T_{\mathrm{v}}$ is calculated from measured temperature $T$ and mixing ratio $m$ :

$T_{\mathrm{v}}=(T+273.15) \cdot\left(1+\frac{0.61 \cdot m}{1000}\right)$.

The virtual temperature is the temperature at which a dry parcel of air would have the same density as the measured moist parcel of air.

\section{Appendix B}

\section{Structure function}

The structure function is a statistic measure to show common variation. The eddy-size distribution of a turbulent flow in the inertial subrange, or the local structure of a turbulent flow, was first and foremost quantified using the (auto-)structure function (Kolmogorov, 1941). See also Bange (2009).

$D_{\phi}(\tau)=\frac{1}{D-\tau} \int_{0}^{D-\tau} \mathrm{d} t[\phi(t+\tau)-\phi(t)]^{2}$,

where $\tau$ is the lag or shift, $\phi$ the physical quantity, $D$ the total length of the time series and $t$ the time.

To simplify the interpretation of structure functions, they can be normalized by dividing by twice the variance of the time series:

$$
\begin{aligned}
\frac{D_{\phi}}{2 \sigma^{2}}= & 0: \text { fully correlated } \\
& 2: \text { non-correlated } \\
& \text { fully anti-correlated. }
\end{aligned}
$$

Acknowledgements. We would like to thank Frank Beyrich and Udo Rummel for the provision of data of all meteorological instruments at the meteorological observatory Lindenberg and their support on-site, Roland Kern for his excellent work on the aircraft, Walter Fichter, Florian Weimer and Alexander Joos at the IFR Stuttgart for their support with the autopilot system and Maximilian Ehrle and Markus Auer for their great job as safety pilots. The measuring equipment would not have been ready to work without the help of Jens Dünnermann and Burkhard Wrenger from the University of Applied Sciences Ostwestfalen-Lippe.

We acknowledge support by Deutsche Forschungsgemeinschaft and Open Access Publishing Fund of Tübingen University.

Edited by: F. X. Meixner

\section{References}

Bange, J.: Airborne Measurement of Turbulent Energy Exchange Between the Earth Surface and the Atmosphere, Sierke Verlag, Göttingen, 174 pp., 2009.

Bange, J., Spieß, T., Herold, M., Beyrich, F., and Hennemuth, B.: Turbulent Fluxes from Helipod Flights above QuasiHomogeneous Patches within the LITFASS Area, Bound.-Lay. Meteorol., 121, 127-151, 2006.

Breitkopf, G. S. W. and Kim, S.: Recovery-Faktor des frontal angeströmten zylindrischen Mantelthermoelementes mit ebener Stirnfläche, Thermo Fluid Dynam, 13, 287-292, 1980.

Daniels, G.: Measurement of gas temperature and the radiation compensating thermocouple, J. Appl. Meteorol., 7, 1026-1035, 1968.

Dias, N., Gonçalves, J., Freire, L., Hasegawa, T., and Malheiros, A.: Obtaining Potential Virtual Temperature Profiles, Entrainment Fluxes, and Spectra from Mini Unmanned Aerial Vehicle Data, Bound.-Lay. Meteorol., 145, 93-111, 2012.

Emeis, S.: Determination of the Surface Sensible Heat Flux From Aircraft Measurements, Betr. Phys. Atmosph., 68, 143-148, 1995. 
Engelbart, D., Steinhagen, H., Görsdorf, U., Lippmann, J., and Neisser, J.: A $1290 \mathrm{MHz}$ profiler with RASS for monitoring wind and temperature in the boundary layer, Beitr. Phys. Atmosph., 69, 63-80, 1996.

Engelbart, D., Steinhagen, H., Görsdorf, U., Neisser, J., Kirtzel, H.J., and Peters, G.: First results of measurements with a newly designed phased-array Sodar with RASS, Meteorol. Atmos. Phys., 71, 71-68, 1999.

Engelbart, D. A. M. and Bange, J.: Determination of BoundaryLayer Parameters using Wind Profiler/RASS and Sodar/RASS in the Frame of the LITFASS-Project, Theor. Appl. Climatol., 73, 53-65, 2002.

Foken, T.: Temperaturmessung mit dünnen Platindrähten, Meteorol. Z., 5, 299-307, 1979.

Friehe, C. A. and Khelif, D.: Fast-response aircraft temperature sensors, 9, 784-795, 1992.

Garratt, J.: The Atmospheric Boundary Layer, University Press, Cambridge, 1992.

Haala, N., Cramer, M., Weimer, F., and Trittler, M.: Performance Test on UAV-Based Photogrammetric Data Collection, vol. 20, in: UAV-g (unmanned aerial vehicle in geomatics) Conference, Zurich, Switzerland, American Meteorological Society, 2011.

Haman, K. E.: A New Thermometric Instrument for Airborne Measurements in Clouds, J. Atmos. Ocean. Tech., 9, 86-90, 1992.

Haman, K. E., Makulski, A., and Malinowski, S. P.: A new ultrafast thermometer for airborne measurements in clouds, J. Atmos. Ocean. Tech., 14, 217-227, 1997.

Harrison, R. and Pedder, M.: Fine wire thermometer for air temperature measurement, Rev. Sci. Instrum., 72, 1539-1541, 2001.

Inverarity, G.: Correcting airborne temperature data for lags introduced by instruments with two-time-constant responses, J. Atmos. Ocean. Tech., 17, 176-184, 2000.

Kaimal, J. C. and Finnigan, J. J.: Atmospheric Boundary Layer Flows - Their Structure and Measurement, Oxford University Press, 289 pp., 1994.

Kolmogorov, A.: The Local Structure of Turbulence in Incompressible Viscous Fluid for Very Large Reynolds Numbers, Dokl. Akad. Nauk SSSR, 30, 299-303, reprint, P. Roy. Soc. Lond. A, 1991, 434, 9-13, 1941.

Lenschow, D.: Probing the Atmospheric Boundary Layer, vol. 1, American Meteorological Society, Boston, MA, USA, 1986.

Luers, J. K.: Temperature Error of the Vaisala RS90 Radiosonde, J. Atmos. Ocean. Tech., 14, 1520-1532, 1997.
Martin, S. and Bange, J.: The Influence of Aircraft Speed Variations on Sensible Heat Flux Measurements by Different Airborne Systems, Bound.-Lay. Meteorol., accepted, 2013.

Martin, S., Bange, J., and Beyrich, F.: Meteorological profiling of the lower troposphere using the research UAV "M2AV Carolo", Atmos. Meas. Tech., 4, 705-716, doi:10.5194/amt-4-705-2011, 2011.

Michalski, L., Eckersdorf, K., and McGhee, J.: Temperature Measurement, John Wiley and Sons Ltd., Chichster, 1991.

Nash, J., Smout, R., Oakley, T., Pathack, B., and Kurnosenko, S.: WMO Intercomparison of High Quality Radiosonde Systems, Tech. rep., WMO Report., available from CIMO, Vacoas, Mauritius, 2-25 February 2005.

Neisser, J., Adam, W., Beyrich, F., Leiterer, U., and Steinhagen, H.: Atmospheric Boundary Layer Monitoring at the Meteorological Observatory Lindenberg as a Part of the 'Lindenberg Column': Facilities and Selected Results, Meteorol. Z. N. F., 11, 241-253, 2002.

Reuder, J., Brisset, P., Jonassen, M., Müller, M., and Mayer, S.: The Small Unmanned Meteorological Observer SUMO: A new tool for atmospheric boundary layer research, Meteorol. Z., 18, 141147, 2009.

Rosemount: Total Temperature Sensors, Tech. Rep. Bulletin 1012, Rosemount Inc., Burnsville, MN, 1986.

Shannon, K. S. and Butler, B. W.: A review of error associated with thermocouple temperature measurement in fire environments, in: Second international wildland fire ecology an fire management congress and fifth symposium on fire and forest meteorology, 16-20 November 2003, Orlando, FL, American Meteorological Society, Boston, MA, p. 7B.4, 2003.

Spiess, T., Bange, J., Buschmann, M., and Vörsmann, P.: First application of the meteorological Mini-UAV "M2AV", Meteorol. Z., 16, 159-169, 2007.

Stull, R.: An Introduction to Boundary Layer Meteorology, Kluwer Acad., Dordrecht, 1988.

van den Kroonenberg, A. C., Martin, T., Buschmann, M., Bange, J., and Vörsmann, P.: Measuring the Wind Vector Using the Autonomous Mini Aerial Vehicle $\mathrm{M}^{2} \mathrm{AV}$, J. Atmos. Ocean. Tech., 25, 1969-1982, 2008.

Wendisch, M. and Brenguier, J.: Airborne Measurements for Environmental Research - Methods and Instruments, vol. 1, WileyVCH, Berlin, 2013. 